\title{
The Case for the Journal's Use of a CG-BY License
}

\author{
Stuart M. Shieber \\ School of Engineering and Applied Sciences, Harvard University, \\ Cambridge, Massachusetts, USA
}

Scholarly writing is different from other writing. Scholars write articles to disseminate their research results without any thought or desire for direct financial recompense. We write so that science and society can benefit from our insights. We write so that our work can be read, used, and reused.

It bears thinking, then, about how best to make our articles available to the world. Under what conditions should we ideally distribute our articles? The issue is especially apposite in the context of an openaccess (OA) journal such as Journal of Language Modelling (JLM). As an open-access journal, a journal for which online distribution is free, $J L M$ is freer to rethink the legal regime under which its articles are distributed, since that distribution does not affect its business model. As a new journal, JLM is in the position to design its policies ab initio, without having to worry about past practice or precedent. As a language-related journal, text is both the medium of communication in $J L M$ and its object of study, bringing front and center the idea of reuse, especially computational reuse, of the text that comprises its articles.

JLM's staff, in consultation with its editorial board, have thought long and hard about the ideal way to achieve the goal of widest possible use and reuse of its articles. In the scholarly communications community, the consensus view, and the view that $J L M$ has settled upon, is to make sure that articles published in the journal are licensed to the world under a broad license that allows every sort of use, subject only to the crucial moral right of proper attribution to the authors. The most direct implementation of that notion is through a Creative Commons license known as CC-BY. 
When authors provide their work under a CC-BY license, they allow anyone to share their work (copy, distribute, and transmit it), to remix the work (to adapt it in various ways), and to make commercial use of the work. However, any use of the work is subject to an attribution requirement: a user must attribute the work properly to the authors, but may not suggest that the authors endorse their use.

Among the many organizations endorsing CC-BY as the license of choice for OA journals are the Open Access Scholarly Publishers Association, SPARC Europe, SURF, and the Directory of Open Access Journals. The SPARC Europe Seal of Approval for journals even requires CC-BY. All the major OA publishers (Public Library of Science, BioMed Central, Hindawi, and many others) have settled on CC-BY as the license to use, as have essentially all OA experts. Community consensus for CC-BY has been expressed by the authors of the Budapest Open Access Initiative's 10th anniversary recommendation in their crisp statement "We recommend CC-BY for all OA journals." (Budapest Open Access Initiative, 2012) Extended arguments for journals' use of CC-BY have been provided by OASPA (Redhead, 2012) and by Michael Carroll (Carroll, 2011).

Some prospective authors may have concerns about the breadth of the CC-BY license. Such worries are important to assuage.

What if someone misuses the material, presenting it in a misleading or inappropriate way, for instance, distributing a version under his or her own name (that is, plagiarizing the work), or providing an inaccurate summary of the work or a bad translation that would reflect badly on me?

Such uses would violate the CC-BY license. Plagiarism directly violates the attribution requirement of the CC-BY license. Misleading statements or implications that the original author provided or endorses a bad summary or translation similarly violate the license. But more importantly, such misuses violate the social norms of all scholarship, norms that have kept such practices in check throughout the modern history of scholarship. Far more than legalistic remedies, norms of behavior are strong incentives not to misuse others' work. Indeed, if moral suasion is insufficient to stop someone from plagiarism or inap- 
propriate attribution, mere legalities of a license are hardly likely to fare better.

What if someone starts selling my articles or running other kinds of businesses making use of my writings? Shouldn't I get paid?

Scholars write for their impact on society, and part of that impact is uptake of their ideas by commercial ventures that improve society through their efforts. Seeing one's work move into the market is a testimony to its importance, not a detriment to be quashed. (As Howard Aiken, the founder of computing research at my own university, has been quoted as saying, "Don't worry about people stealing your ideas. If your ideas are any good, you'll have to ram them down people's throats.")

Keep in mind that although CC-BY allows for commercial reuse, such reuses would need to be something more than simply reselling content. When articles are available for free as in an OA journal like $J L M$, there is essentially no market for pure resale of the articles. Any commercial venture using CC-BY-licensed articles as a part of the business process would need to add value to those raw materials, and insofar as it does so, there would seem to be no argument against legitimate compensation of the business for its efforts in providing that value. If value is added, why not allow recouping of expenses and profit? The knee-jerk reaction against commercial use of scholarly articles has been termed "profit-spite" by Jan Velterop. The sentiment that "if I can't make money off of my article, no one should" may be appealing at first blush, but collapses under an understanding of the scholarly enterprise.

Some of this reaction may be a natural result of popular sentiment against perceived gouging by certain publishers of subscription journals. But the reaction to problems in the subscription journal market is not to blame the publishers, but rather to blame the cause of the systemic market dysfunction, monopolistic ownership. CC-BY eliminates that fundamental problem. When the raw materials for a business are freely available, it's hard for a business to gouge in selling its valueadded products and services, because any potential competitor has the same free access to those raw materials. 
But if someone reuses my article in some way, shouldn't they be required to at least share the results with the community for free?

Licenses like the "copy-left" license that requires "sharing alike" are appropriate for many situations, especially open-source software projects, where individual modifications of a single item (a software application, say) by themselves can have major value that could otherwise be locked up. But for scholarly articles, any added value would typically come from the ability to aggregate large volumes of articles and extract value from the aggregation. Requiring share-alike would disallow such aggregations, especially when the aggregation includes materials under more restrictive licenses. The overhead of tracking these combined licenses has led many, even in the open-source software community, to eschew share-alike licenses.

I'm proud to be associated with a journal that has made the right choice in ensuring that its articles can be used in the most open and appropriate manner. Journals like $J L M$ that act in the best interest of our community of scholars deserve our support.

\section{REFERENCES}

BudAPEST Open ACCESS INitiative (2012), Ten years on from the Budapest Open Access Initiative: setting the default to open, URL http://wWw. opensocietyfoundations . org/openaccess/boai-10-recommendations.

Michael W. CARroll (2011), Why Full Open Access Matters, PLoS Biology, 9(11):e1001210, doi:10.1371/journal.pbio.1001210, URL http://dx . doi . org/10.1371\%2Fjournal . pbio . 1001210.

Claire REDHEAD (2012), Why CC-BY?, Open Access Scholarly Publishers Association Blog, URL http: //oaspa.org/why-cc-by/.

This work is licensed under the Creative Commons Attribution 3.0 Unported License. http://creativecommons.org/licenses/by/3.๑/

(c) BY 